Voix et Images

volxetimages

\title{
Largillierre ou l'art de rater le train en beauté
}

\section{Jean-Pierre Duquette}

Volume 7, numéro 2, hiver 1982

Michel Tremblay

URI : https://id.erudit.org/iderudit/200329ar

DOI : https://doi.org/10.7202/200329ar

Aller au sommaire du numéro

Éditeur(s)

Les Presses de l'Université du Québec

ISSN

0318-9201 (imprimé)

1705-933X (numérique)

Découvrir la revue

Citer cet article

Duquette, J.-P. (1982). Largillierre ou l'art de rater le train en beauté. Voix et Images, 7(2), 401-403. https://doi.org/10.7202/200329ar d'utilisation que vous pouvez consulter en ligne.

https://apropos.erudit.org/fr/usagers/politique-dutilisation/ 
Art

\title{
Largillierre ou l'art de rater le train en beauté
}

\author{
par Jean-Pierre Duquette
}

Une fois retombées les brèves et maigres agitations qui ont marqué l'ouverture de l'exposition Largillierre en septembre 1981, on se dit que tous ces énervements, encore une fois, sentaient beaucoup la province. On a vite fait, dans Landerneau, de crier au scandale pour des broutilles et de poser au martyr. Tout, cette fois, tournait autour d'un malheureux demi-million de dollars jeté par les fenêtres du musée de la rue Sherbrooke pour un peintre de vingt-quatrième ordre, tandis que les artistes du cru sont si mal traités au Québec en général, et par la dite institution en particulier. Le ministre des Affaires culturelles a dô frémir d'appréhension en recevant le télégramme, laconique autant que péremptoire, lui enjoignant de faire maison nette dans l'administration du Musée des Beaux-Arts. Les expéditeurs étaient en l'occurrence cinq groupes d'artistes professionnels, ceux-là même qui avaient organisé une mini-manif à l'entrée du musée, pancartes au vent sous une petite pluie sournoise, dans les décombres des trottoirs défoncés pour cause d'embellissement urbain.

Certes, la maladie qu'ont attrapée les conservateurs, ces récentes années, de mettre sur pied des super-shows pour attirer les amateurs du monde entier, via tous les ticketrons crépitant de par les capitales, a quelque chose de passablement agaçant. C'est au musée qui verra à ses portes les queues les plus longues, les affluences les plus vastes, les retombées les plus juteuses grâce à la vente d'affiches, de tee-shirts, de badges et autres gadgets pour demeurés. Sans parler des reproductions, bien entendu. On nous assure du reste que ces spectacles à grand déploiement sont désormais terminés, même dans les grandes institutions: les hausses de toute sorte (transport des œuvres, assurances, recherches, production de catalogues épais comme des briques) font que le jeu n'en vaut décidément plus la chandelle. Et puis, il faut miser juste. Picasso au MOMA, van Gogh à Toronto, va toujours: le calendrier et la carte postale les ont propulsés jusque dans les foyers les moins pourvus de "culture artistique», comme dit l'autre. Mais un portraitiste mondain? du XVIIle siècle? célèbre en son temps, on veut bien, néanmoins tombé dans l'oubli depuis belle lurette? 
et dont plusieurs - et pas des moindres - ne connaissaient même pas le nom? C'était aller droit au désastre. De fait, même si les salles n'ont pas toujours été totalement désertes, il y a rarement eu émeute au portillon. Cela veut-il dire, par contre, que le MBA ne devait pas monter cette exposition?

Il s'agit d'un des portraitistes français les plus significatifs de son temps. Qu'on juge pompiers son style, l'attitude des modèles, le décor et les brimborions, cela ne change rien au fait que Largillierre compte parmi les artistes de premier rang en France et en Europe à la fin du XVII e siècle et au début du XVIII e. C'est peut-être du reste cette grandiloquence, les bouillonnés, les drapés extravagants, tout l'appareil baroque et théâtral, qui servent d'abord de prétexte à ceux qui regardent cette peinture par le gros bout de la lorgnette: ce n'est certes pas là "notre" XVIIle siècle, en Nouvelle-France. Loin du grand faste de Versailles, notre première peinture et les portraits de notre petite aristocratie ne pouvaient être qu'un pâle reflet - et combien maladroit - des gloires métropolitaines, tout émouvant qu'il fût dans sa naïveté et son absence de prétention. Parions qu'une exposition aussi spectaculaire, mais sur la peinture religieuse de la même époque (voir la chapelle des Ursulines à Québec), aurait déclenché moins de fureur chez la gent artiste... Passons sur l'accusation d'académisme : les accessoires et les déguisements à l'Antique sont, au départ, partie intégrante de la peinture officielle des débuts de Largillierre. Ces portraits étaient bien, dans leur pompe et leurs boursouflures, des signes, des annonces d'appartenance (ou de désir d'appartenance) à un monde pour qui le paraître était (presque) déjà l'être. Est-ce dire que Largillierre n'a fait que de la production à la chaîne, visages interchangeables sur fonds tout apprêtés? Il est arrivé, bien sûr, qu'il travaille de cette façon. Mais en revanche, quelle maîtrise des coloris, quel sens de la mise en place, quelle vérité profonde dans les regards. C'est dans des portraits aussi peu «spectaculaires» que celui de Voltaire jeune qu'on mesure toute la finesse et l'art du peintre. Ici, aucune draperie somptueuse, pas de cascades savantes de velours ou de soie moirée: dans l'ovale du cadre, un homme, à mi-corps, vous regarde dans les yeux, un sourire à peine esquissé, déjà l'air d'en penser long sur tout cela.

Du reste, ne travaillant pas uniquement pour la noblesse, Largillierre en viendra peu à peu à portraiturer la grande bourgeoisie, les gens de robe et de banque. En même temps que l'appareil social commence lentement à bouger, on s'éloigne peu à peu du portrait d'apparat, du style de cour Grand Siècle. Avec Nattier et Tocqué, par exemple, la génération suivante nous donnera des portraits beaucoup plus détendus, moins guindés, plus souples. Largillierre est l'un de ceux qui ont ouvert la voie à cette simplicité accrue, à ce naturalisme plus proche de la vie, de la quotidienneté.

Outre la révélation d'un grand peintre, cette exposition aura été l'occasion de recherches approfondies, et l'un des fruits les plus importants en 
demeurera précisément le catalogue, paru avec un retard difficilement excusable, comme d'habitude. Cet ouvrage de près de quatre cents pages, œuvre collective de spécialistes québécois, français, américains, soviétiques, constituera une somme indispensable sur Largillierre et son temps, sur l'art du portrait aux XVII e et XVIII e siècles, sur l'influence du peintre sur ceux qui viendront après lui. Comme l'a affirmé très justement le directeur du MBA, Jean Trudel, un musée comme celui-ci, même avec des budgets relativement modestes, doit consacrer une part de ses activités et de ses compétences à la recherche, aller à la découverte, mettre en lumière des périodes ou des artistes qui ont été à tort négligés. Tous les petits maîtres ne sont évidemment pas des mines d'éblouissement - et de toute façon, encore une fois, Largillierre est loin d'être un peintre mineur, comme certains l'ont affirmé un peu vite. Répondant au tintoin des protestataires, les spécialistes venus de France, d'Angleterre, d'Espagne, des ÉtatsUnis et d'URSS ont signé une mise au point sur la valeur de cette entreprise : "Un musée vivant et soucieux de culture vivante ne saurait se contenter d'offrir au public des œuvres dont chacun a, ou peut avoir, chez soi albums ou reproductions. II doit contribuer à la mise en valeur du patrimoine artistique, lequel ne connaît pas de frontières. Il doit éclairer, pour les créateurs d'aujourd'hui comme pour tous ceux qui s'intéressent à l'art, la création du passé dans toute sa variété et sa richesse. " On pourra toujours déplorer le coût de cette entreprise, le fait qu'aucun autre musée n'ait été intéressé à "acheter" l'exposition. II reste qu'une telle manifestation, même largement déficitaire, honore le MBA et les membres de son conseil d'administration qui ont soutenu jusqu'au bout l'initiative de la conservatrice à qui nous devons cette redécouverte, Myra Nan Rosenfeld. 